\title{
A cannabinoid mechanism in relapse to cocaine seeking
}

\author{
Taco J. De Vries ${ }^{1}$, Yavin Shaham ${ }^{2}$, Judith R. Homberg ${ }^{1}$, Hans Crombag ${ }^{2}$, \\ KarianNe SCHUURMAN ${ }^{1}$, JEANINE DiEBEN ${ }^{1}$, LOUK J.M.J. VANDERSCHUREN ${ }^{1} \&$ \\ ANTON N.M. SCHOFFELMEER ${ }^{1}$
}

Treatment of cocaine addiction is hampered by high rates of relapse even after prolonged drug abstinence. This relapse to compulsive cocaine use can be triggered by re-exposure to cocaine ${ }^{1}$, by re-exposure to stimuli previously associated with cocaine $^{2}$ or by exposure to stress ${ }^{3}$. In laboratory rats, similar events reinstate cocaine seeking after prolonged withdrawal periods ${ }^{4-6}$, thus providing a model to study neuronal mechanisms underlying the relapse to cocaine. The endocannabinoid system has been implicated in a number of neuropsychiatric conditions, including drug addiction ${ }^{7,8}$. The active ingredient of marijuana, $\Delta$ 9-tetrahydrocannabinol, activates the mesolimbic dopamine (DA) reward system ${ }^{9,10}$ and has rewarding effects in preclinical models of drug abuse ${ }^{8,11,12}$. We report here that the synthetic cannabinoid agonist, HU210 (ref. 13), provokes relapse to cocaine seeking after prolonged withdrawal periods. Furthermore, the selective CB1 receptor antagonist, SR141716A (ref. 14), attenuates relapse induced by re-exposure to cocaine-associated cues or cocaine itself, but not relapse induced by exposure to stress. These data reveal an important role of the cannabinoid system in the neuronal processes underlying relapse to cocaine seeking, and provide a rationale for the use of cannabinoid receptor antagonists for the prevention of relapse to cocaine use.

To study the role of the cannabinoid system in relapse to cocaine seeking, we used a 'reinstatement model' ${ }^{15}$ in which we tested rats for relapse behavior induced by drug or non-drug stimuli, following extinction of the drug-taking behavior that was previously maintained by cocaine. This preclinical model simulates the human situation in which cocaine addicts relapse to compulsive drug use after prolonged withdrawal periods ${ }^{1-3}$.

First we evaluated whether activation of cannabinoid receptors by the synthetic cannabinoid agonist, HU210, would reinstate cocaine seeking after a prolonged withdrawal period. In daily two-hour sessions, rats were trained to nose poke for intravenous (i.v.) cocaine self-administration. Subsequently, responding for cocaine was extinguished in the absence of the drug for at least 14 days. Rats were then tested under extinction conditions (that is, cocaine was not available) for reinstatement of drug seeking induced by systemic injections of HU210 or its vehicle. We found that HU210 reinstates cocaine seeking in a dose-dependent manner (Fig. 1a). This effect was reversed with the selective CB1 receptor antagonist SR141716A. When given alone, the cannabinoid antagonist did not modify baseline response rates (Fig. $1 b$ ). These data indicate that the 'relapse' provoking effect of HU210 is mediated by CB1 receptors. The biphasic dose-effect curve with HU210 observed here is similar to that ob- served in previous studies on the behavioral effects of this and related compounds $s^{8,12}$.

There is a general agreement that the central effects of the endocannabinoids anandamide and 2-arachidonylglycerol are mediated by CB1 receptors ${ }^{8,13}$. Therefore, the CB1 receptor may represent a target for a pharmacological intervention to prevent relapse to cocaine seeking. To test this possibility, we determined whether SR141716A would attenuate cocaine-seeking behavior. First, we determined the effect of SR141716A on reinstatement of cocaine seeking induced by non-contingent (priming) injections of cocaine. Rats were trained for cocaine self-administration and then underwent extinction for at least 14 days. On the test days, rats received a computer-controlled, priming infusion of cocaine $(1.0 \mathrm{mg} / \mathrm{kg}$, i.v.). Infusions of cocaine reinstated responding on the previously cocaine-paired hole, an effect that was attenuated by SR141716A (Fig. 2a).

Next, we studied reinstatement of cocaine seeking induced by re-exposure to cocaine-associated cues, that is, a houselight that previously predicted drug availability and a 15-second click/light discrete cue that was associated with cocaine infusions. We trained rats to nose poke for cocaine infusions for two weeks. During subsequent extinction training, which lasted for three weeks, the overhead light was not turned on at the start of each session and nose poking had no consequences (cocaine infusions and the click/light signal were not presented). On the test days, the overhead light previously predicting cocaine availability was turned on at the start of the session. In addition, every fifth nose poke resulted in the presentation of the click/light signal (previously paired with cocaine infusions), but not in cocaine delivery. Re-exposure to the cocaine-paired cues reliably reinstated extinguished responding on the previously active hole, an effect that was attenuated by SR141716A in a dose-dependent manner (Fig. $2 b$ ). Thus, these data indicate that activation of the CB1 receptor is involved in relapse to cocaine seeking induced by re-exposure to cocaine or cocaine-associated cues.

Another well-documented stimulus to trigger relapse-like behavior in rats is brief exposure to a mild foot-shock stressor ${ }^{5}$. We therefore tested the effect of SR141716A on stress-induced reinstatement of cocaine seeking. Exposure to a 10-min intermittent foot-shock stressor reinstated operant responding (Fig. 2c). In contrast to the data above, however, this effect was not attenuated by SR141716A. Thus, activation of the CB1 receptor does not appear to contribute to relapse induced by an environmental stressor.

The doses of SR141716A used here are within the range previously shown to block neurochemical and behavioral effects of cannabinoids, without affecting spontaneous motor activity ${ }^{8,12}$. 

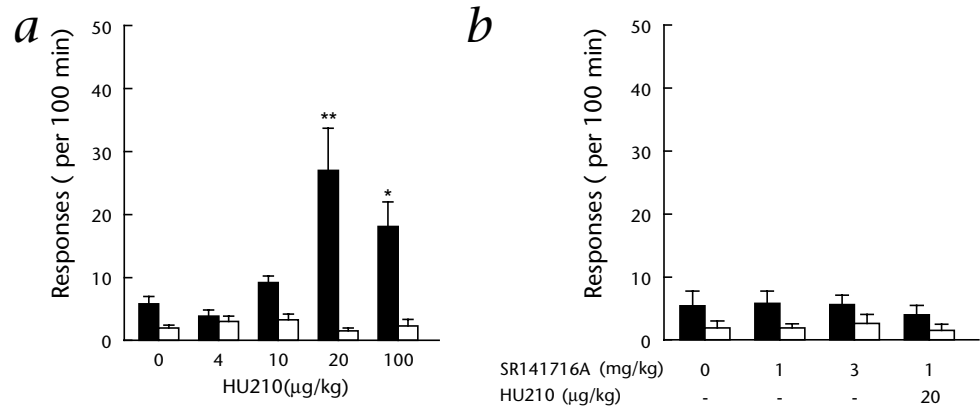

Fig. 1 The potent cannabinoid receptor agonist HU210 reinstates cocaine-seeking behavior. $\boldsymbol{a}$, In animals withdrawn from i.v. cocaine self-administration during daily extinction sessions for $3 \mathrm{wk}$, reinstatement tests were performed during which animals were primed with s.c. injections of HU210. HU210 produces a dose-dependent reinstatement of responding in the previously cocaine-paired hole $(\boldsymbol{\square}) . \mathrm{F}[1,54]=9.77, P<0.01$, by analysis of variance; ${ }^{*}, P<0.05 ;{ }^{* *}, P<0.01 ; n=7-9$ per dose. HU210 does not alter the number of responses in the inactive hole $(\square)$, a measure of non-specific activity and/or response generalization $(P>0.1)$. $\boldsymbol{b}$, SR141716A has no effect on baseline nose poke responding, but the $1.0 \mathrm{mg} / \mathrm{kg}$ dose blocks HU210-induced reinstatement of responding for cocaine ( $n=8$ per dose).

However, to further determine the specificity of the effect of SR141716A on cue- and cocaine-priming-induced reinstatement of cocaine seeking, we studied the effect of the CB1 receptor antagonist on the maintenance of cocaine self-administration and on the self-administration of a 10\% sucrose solution, a potent non-drug reinforcer in rats. SR141716A had no effect on cocaine or sucrose selfadministration behavior (Fig. 3). The lack of effect of SR141716A on the maintenance of cocaine self-administration corroborates previous reports ${ }^{11,16}$ indicating that blockade or the absence of the CB1 receptor does not alter the primary rewarding effects of cocaine. However, the results with sucrose-trained rats are different from pre- vious reports in which SR141716A decreased consumption of sucrose ${ }^{12}$. Procedural differences, such as limited versus free access to sucrose and the measurement of lever-pressing behavior versus drinking behavior may account for these discrepant results.

We have found that a cannabinoid agonist precipitates relapse to cocaine seeking, whereas a CB1-receptor antagonist selectively reduces relapse behavior induced by cocaine or by conditioned cocaine cues, but not relapse induced by an environmental stressor. Previous studies indicate that the mesolimbic DA system is involved in reinstatement induced by re-exposure to cocaine or cocaine-associated cues $^{15}$. For example, cocaine seeking induced by cocaine itself or by cocaine cues is attenuated by DA receptor antagonists ${ }^{17}$. In addition, environmental cues previously paired with cocaine self-administration elevate DA levels in the amygdala ${ }^{6,18}$ and nucleus accumbens $(\mathrm{NAc})^{6}$, terminal regions of the mesolimbic DA system. Moreover, increasing DA transmission by morphine infusions into the ventral tegmental area (VTA) or by amphetamine infusions in the NAc reinstates drug-taking behavior ${ }^{15}$. These observations are of particular interest because there is evidence that the release of the endogenous cannabinoid anandamide in the striatal complex ${ }^{19}$ is mediated in part through activation of local DA D2-like receptors ${ }^{20}$. Therefore, levels of DA elevated by cocaine or cocaine cues may trigger the release of endocannabinoids. Thus, endocannabinoids or synthetic cannabinoid agonists such as HU210 may mediate relapse to cocaine seeking by acting on CB1 receptors downstream from the DA synapse. Alternatively, cannabinoids may act upstream from DA synapses, by increasing the release of DA in terminal regions of the mesolimbic DA system ${ }^{9,10,21}$, which in turn induces reinstatement of cocaine seeking ${ }^{15}$. However, the possibility that activation of CB1 receptors upstream of the DA synapse medi- $a$

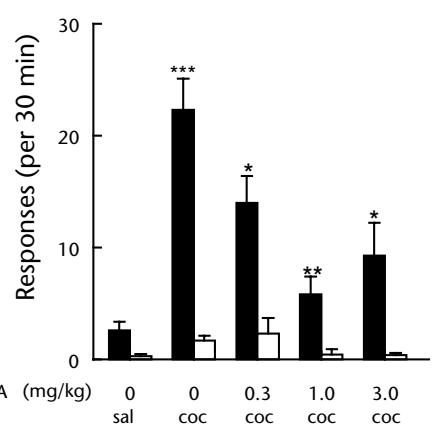

$b$

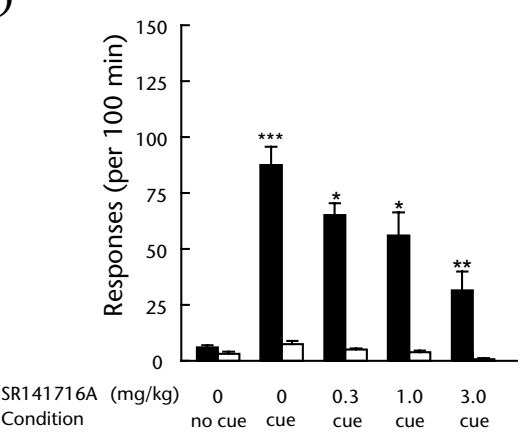

C

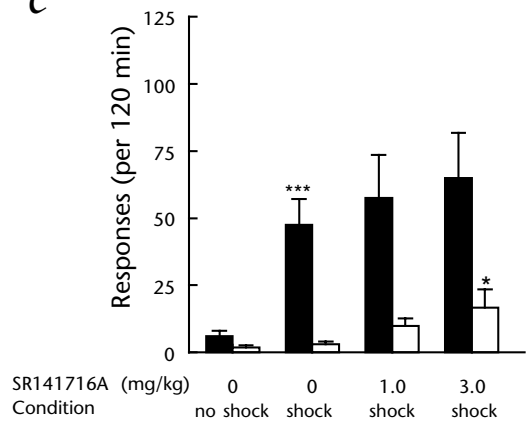

Fig. 2 The cannabinoid CB1 receptor antagonist, SR141716A, attenuates cocaine- and cue-induced reinstatement of cocaine-seeking behavior, but not stress-induced reinstatement. a, Effect of SR141716A on cocaine-induced reinstatement of responding in the previously cocaine-paired hole (匹) and in the inactive hole ( $\square$ ). Computer-controlled i.v. infusion of cocaine (' ${ }^{\prime} \mathrm{c}^{\prime}, 1.0 \mathrm{mg} / \mathrm{kg}$ ) selectively reinstates responding in the previously cocaine-paired hole in animals in which responding for cocaine was extinguished for at least $14 \mathrm{~d}$. $F[1,42]=87.7, P<0.001 ; * \star *, P<0.001)$. Pretreatment with SR141716A attenuates cocaine-induced responding. $\mathrm{F}[1,44]=21.2, P<0.001 ;{ }^{*}, P<0.02 ;{ }^{* *}, P<0.001 ; n=7-9$ per dose). Responding in the inactive hole is not different from the saline i.v. control condition ('sal'). b, Effect of SR141716A on cue-induced reinstatement of responding in the previously cocaine-paired hole ( $\square)$ and in the inactive hole ( $\square$ ). Rats are tested after $21 \mathrm{~d}$ of extinction of the cocaine self-adminis- tration behavior. Re-exposure to cocaine-associated cues reinstates responding in the previously cocaine-paired hole. $F[1,41]=97.4, P<0.001$ by ANOVA; $\left.{ }^{* *}, P<0.001\right)$. In the presence of SR141716A, the increase of responding is attenuated. $F[1,58]=13.94, P<0.001 ;{ }^{*}, P<0.05 ;{ }^{* *}, P<$ $0.01 ; n=9-12$ per dose. Responding in the inactive hole is not different from the 'no cue' condition. c, Effect of SR141716A on foot-shock-induced reinstatement of responding on the previously cocaine-paired lever ( $\square$ ) and on the inactive lever $(\square)$. Exposure to $10 \mathrm{~min}$ of intermittent foot-shock reinstates responding on the previously cocaine-paired lever. $F[1,18]=32.8$, $P<0.001 ;{ }^{* * *}, P<0.01$. This effect of the stressor is not attenuated by pretreatment with SR141716A ( $P>0.1 ; n=9-10$ per dose). However, the cannabinoid antagonist somewhat increases responding on the inactive lever in the shock condition $\left({ }^{*}, P<0.05\right)$. SR141716A by itself does not produce any changes in basal responding in the 3 different experiments. 
$a$

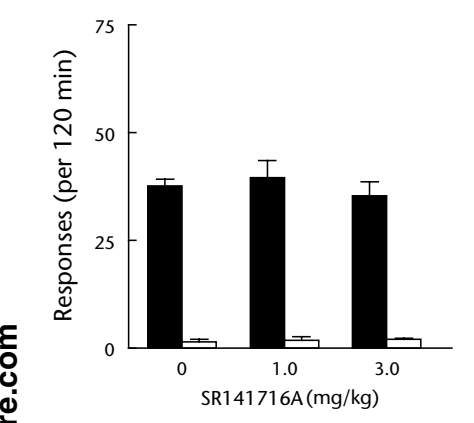

$b$

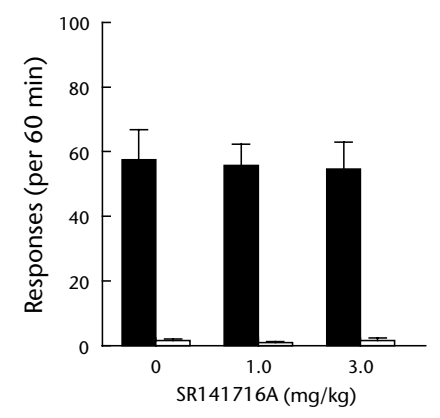

ates the effect of the CB1 antagonist on relapse to cocaine is less likely. Thus, whereas cannabinoid agonists were found to increase DA release in the NAc via their indirect action on $\mu$-opioid receptors in the cell-body region of the VTA (ref. 10), blockade of opioid receptors had no effect on cocaine-priming-induced reinstatement ${ }^{22}$. Alternatively, the effect of SR141716A may be the result of the reported inverse agonistic properties of this compound ${ }^{8,23}$. This explanation, however, is not likely to account for the data here. The inverse agonist-like effect of SR141716A requires the presence of constitutively activated CB1 receptors and should therefore be most pronounced under basal conditions, when endogenous agonist levels are low. Here, however, SR141716A by itself had no effect on behavior under basal conditions (Fig. 1b). A recent study in humans, in which SR141716A was found to attenuate the effects of smoked marijuana, did not report any physiological or psychological effects of the antagonist when given alone $e^{24}$.

We have found that the cannabinoid receptor antagonist does not alter the primary rewarding effects of cocaine. This specific effect on relapse to cocaine seeking induced by cocaine and cocaine cues, but not on stable drug self-administration behavior, is in agreement with recent evidence suggesting that dissociable neuronal mechanisms underlie these behaviors ${ }^{25,26}$. For example, it was found that while D1-like receptor agonists are self-administered by laboratory animals, they do not induce reinstatement of cocaine seeking and even attenuate reinstatement induced by cocaine priming injection $\mathrm{s}^{4,17,27}$. In addition, recent studies indicate that while the basolateral amygdala is involved in the secondary rewarding effects of conditioned drug cues, the integrity of the $\mathrm{NAc}$ is essential for responding for cocaine itself $^{26,28}$. Finally, the lack of effect of SR141716A on reinstatement of cocaine seeking induced by a foot-shock stressor is in agreement with recent reports on pharmacological dissociation between stress- and druginduced reinstatement of drug seeking. For example, stress-induced reinstatement of heroin seeking is relatively insensitive to DA receptor antagonists ${ }^{5}$, whereas, as mentioned above, the DA system is critically involved in drug-priming and cue-induced reinstatement ${ }^{15}$. In contrast, brain corticotrophin-releasing factor (CRF) and noradrenaline systems contribute to stress-, but not drug-induced reinstatement of heroin and cocaine seeking ${ }^{5}$.

Recent studies in our laboratory show that cannabinoid receptors are also involved in relapse behavior in rats with a history of heroin self-administration. The cannabinoid receptor agonist, HU210, was found to reinstate heroin seeking following prolonged withdrawal periods, while cue-induced reinstatement of heroin seeking was attenuated by the CB1 receptor antagonist, SR141716A (T.J.D.V., unpublished data). We therefore propose that the 'anti-relapse' effect of SR141716A provides

Fig. 3 SR141716A does not affect the primary rewarding effects of cocaine or sucrose. $\boldsymbol{a}$, In animals trained to self-administer cocaine $(0.5 \mathrm{mg} / \mathrm{kg})$ on an FR-5 schedule of reinforcement for $2 \mathrm{~h} / \mathrm{d}$, pre-treatment with SR141716A ( 1.0 and $3.0 \mathrm{mg} / \mathrm{kg} ; n=9$ per dose) does not affect the number of cocaine infusions ( $\square$ ) obtained (baseline responding was $40.1 \pm 3.4$ infusions per $2 \mathrm{~h}$ ), nor the number of inactive responses $(\square)$. $\boldsymbol{b}$, In animals trained to orally self-administer a $10 \%$ sucrose solution for $1 \mathrm{~h} / \mathrm{d}$, pre-treatment with SR141716A ( $n=8$ per dose) does not alter the number of sucrose infusions (घ) obtained, nor the number of inactive lever responses $(\square)$.

a rationale for the use of cannabinoid receptor antagonists for the prevention of relapse in abstinent drug addicts.

\section{Methods}

Subjects. Male Wistar (Harlan, the Netherlands) or Long-Evans (Charles River, Massachusetts) rats (300-400 g) were used. Catheters were implanted into the jugular vein as described ${ }^{4,5}$. Procedures were approved by the local Animal Care and Use Committees and were done in accordance with NIH guidelines.

Drugs. SR141716A (National Institute of Mental Health's Chemical Synthesis and Drug Supply Program, Bethesda, Maryland) and HU210 (Tocris, Bristol, UK) were dissolved in ethanol, Tween 80 and sterile saline (ratio 1:1:18) and administered subcutaneously (s.c.). Cocaine $\mathrm{HCl}$ (OPG, Amsterdam, The Netherlands; NIDA, Baltimore, Maryland) was dissolved in saline.

HU210-induced reinstatement. Rats were trained to self-administer cocaine in operant chambers under a fixed-ratio 1 (FR-1) schedule of reinforcement (each nose poke is reinforced) for 2 -h daily sessions. Responding in the active hole resulted in the infusion of cocaine $(0.5 \mathrm{mg} / \mathrm{kg})$. A houselight was turned on during the daily sessions. For $15 \mathrm{~s}$ after each cocaine infusion, the houselight was turned off and the infusions were accompanied by a click of an electrical switch. Responding in the inactive hole had no consequences. When rats acquired stable responding (usually within 5 sessions), the response requirement was gradually increased to an FR-5 schedule of reinforcement (every 5 th response led to cocaine infusion). Subsequently, extinction sessions (100 min), during which nose-poke responses had no consequences, were conducted daily for at least 14 days or until the rats reached an extinction criterion of less than 10 responses on the previously active hole. On the test days (conducted in a counterbalanced order under extinction conditions), rats were given s.c. injections of the cannabinoid agonist HU210 or its vehicle $10 \mathrm{~min}$ before the sessions. In an additional experiment, the effect of the CB1 receptor antagonist SR141716A alone (30 min pre-treatment time) on HU210-induced cocaine seeking was evaluated.

Cocaine-induced reinstatement. Rats were trained for cocaine self-administration under an FR-1 schedule of reinforcement for 10-12 d. During subsequent daily 60-min extinction sessions (14 d), responding had no consequences. $30 \mathrm{~min}$ after the onset of each extinction session, rats received a computer-controlled infusion of saline (i.v.). On the test days, the conditions were identical, but rats received a computer-controlled infusion of cocaine $(1.0 \mathrm{mg} / \mathrm{kg}$, i.v.). Nose poking, in the absence of previously cocaine-paired cues (the click/light signal) and without receiving further cocaine infusions, served as a measure of relapse behavior. Vehicle or SR141716A injections were given, in a counterbalanced order, $30 \mathrm{~min}$ before the test sessions, which were conducted every $48-72 \mathrm{~h}$.

Cue-induced reinstatement. Rats were trained to self-administer cocaine as described above. During the extinction phase ( $21 \mathrm{~d}$ ), cocaine was not available and the cues previously associated with cocaine availability (the houselight) or cocaine infusions (the tone/light signal) were not presented. On the test day, rats were tested under extinction conditions, but this time the houselight was turned on. In addition, every 5 th nose poke was accompanied by the click of the electrical switch and the turning off of the houselight for $15 \mathrm{~s}$. Vehicle or SR141716A was given, in a counterbalanced order, $30 \mathrm{~min}$ before the 100-min test sessions, which were conducted every $48-72 \mathrm{~h}$.

Stress-induced reinstatement. Rats were chronically housed in the operant chambers and were trained to self-administer cocaine for $12 \mathrm{~d}(2 \mathrm{~h} / \mathrm{d})$. Each 
response on one (active) lever resulted in the delivery of cocaine $(0.5 \mathrm{mg} / \mathrm{kg})$ and the illumination of a cue light for $15 \mathrm{~s}$. Presses on the other (inactive) lever were also recorded. The sessions began with the illumination of the houselight and the insertion of the active lever. At the end of the sessions, the houselight was turned off and the active lever was retracted. During the extinction phase (14 d), the conditions were the same as during self-administration training except that the cocaine syringes were removed. Tests for foot-shock-induced reinstatement were conducted during 4 daily sessions, which were separated by $48 \mathrm{~h}$. The intermittent foot-shock stressor $(0.6 \mathrm{~mA}$, $0.5 \mathrm{~s}$ 'on' period; a mean 'off' period of $40 \mathrm{~s}$ ) was given just prior to the test sessions. Vehicle and SR141716A were given, in a counterbalanced order, 30

Cocaine self-administration. The training conditions were identical to those described above. After stable cocaine self-administration was obtained on an FR-5 schedule, the effect of SR141716A (administered 30 min before the session) or its vehicle on the drug-reinforced behavior was evaluated. Tests were conducted every $2 \mathrm{~d}$ and the order of the administration of the SR141716A doses was counterbalanced.

Sucrose self-administration. The self-administration chambers were fitted with liquid drop receptacles. To facilitate acquisition of responding, rats were water deprived for $21 \mathrm{~h}$ prior to the first session. Rats were trained to lever press for a $10 \%$ sucrose solution $(0.2 \mathrm{ml}$ per reinforcer delivery; FR-1 schedule) for $60 \mathrm{~min} / \mathrm{d}$. After stable lever responding was obtained, the effects of SR141716A or its vehicle (given in a counterbalanced order every $2 \mathrm{~d}$ ) on sucrose self-administration were determined.

\section{Acknowledgments}

We thank R. Binnekade, H. Raasø and J. Yap for technical support; and J. Grimm and E. Gardner for helpful comments. Part of this study was supported by funds from intramural research program, NIDA/NIH.

\section{RECEIVED 28 JUNE; ACCEPTED 28 AUGUST 2001}

1. de Wit, H. Priming effects with drugs and other reinforcers. Exp. Clin. Psychopharmacol. 4, 5-10 (1996).

2. Childress, A.R., Ehrman, R., Rohsenow, D.J., Robbins, S.J. \& O'Brien, C.P. in Substance Abuse: A Comprehensive Textbook (eds. Lowinson, J.W., Luiz, P., Millman, R.B. \& Langard, G.) 56-69 (Williams and Wilkins, Baltimore, Maryland, 1992).

3. Sinha, R., Catapano, D. \& O'Mally, S. Stress-induced craving and stress responses in cocaine dependent individuals. Psychopharmacology 142, 343-351 (1999).

4. De Vries, T. J., Schoffelmeer, A.N.M., Binnekade, R. \& Vanderschuren, L.J.M.J. Dopaminergic mechanisms mediating the incentive to seek cocaine and heroin following long-term withdrawal of IV drug self- administration. Psychopharmacology 143, 254-260 (1999).

5. Shaham, Y., Erb, S. \& Stewart, J. Stress-induced relapse to heroin and cocaine seeking in rats: a review. Brain Res. Rev. 33, 13-33 (2000)

6. Weiss, F. et al. Control of cocaine-seeking behavior by drug-associated stimuli in rats:
Effects on recovery of extinguished operant-responding and extracellular dopamine levels in amygdala and nucleus accumbens. Proc. Nat. Acad. Sci. USA 97, 4321-4326 (2000).

7. Piomelli, D., Giuffrida, A., Calignano, A. \& de Fonseca, F. R. The endocannabinoid system as a target for therapeutic drugs. Trends Pharmacol. Sci. 21, 218-224 (2000)

8. Ameri, A. The effects of cannabinoids on the brain. Prog. Neurobiol. 58, 315-348 (1999).

9. Chen, J.P., Paredes, W., Lowinson, J.H. \& Gardner, E.L. Strain-specific facilitation of dopamine efflux by $\Delta 9$ - tetrahydrocannabinol in the nucleus accumbens of rats: an in vivo microdialysis study. Neurosci. Lett. 129, 136-180 (1991).

10. Tanda, G., Pontieri, F. E. \& Di Chiara, G. Cannabinoid and heroin activation of mesolimbic dopamine transmission by a common $\mu-1$ opioid receptor mechanism. Science 276, 2048-2050 (1997).

11. Tanda, G., Munzar, P. \& Goldberg, S.R. Self-administration behavior is maintained by the psychoactive ingredient of marijuana in squirrel monkeys. Nature Neurosci. 3, 1073-1074 (2000).

12. Chaperon, F. \& Thiebot, M. H. Behavioral effects of cannabinoid agents in animals. Critical Rev. Neurobiol. 13, 243-281 (1999).

13. Mechoulam, R., Fride, E. \& Di Marzo, V. Endocannabinoids. Eur. J. Pharmacol. 359, 1-18 (1998).

14. Rinaldi-Carmona, M. et al. SR141716A, a potent and selective antagonist of the brain cannabinoid receptor. FEBS Lett. 350, 240-244 (1994).

15. Stewart, J. Pathways to relapse: The neurobiology of drug- and stress-induced relapse to drug-taking. J. Psychiatry Neurosci. 25, 125-136 (2000).

16. Cossu, G. et al. Cannabinoid CB1 receptor knockout mice fail to self-administer morphine but not other drugs of abuse. Behav. Brain Res. 118, 61-65 (2001).

17. Khroyan, T.V., Barrett-Larimore, R.L., Rowlett, J.K. \& Spealman, R.D. Dopamine D1and D2-like receptor mechanisms in relapse to cocaine-seeking behavior: Effects of selective antagonists and agonists. J. Pharmacol. Exp. Ther. 294, 680-687 (2000).

18. Tran-Nguyen, L.L. et al. Time-dependent changes in cocaine-seeking behavior and extracellular dopamine levels in the amygdala during cocaine withdrawal. Neuropsychopharmacol. 19, 48-59 (1998).

19. Di Marzo, V. et al. Enhancement of anandamide formation in the limbic forebrain and reduction of endocannabinoid contents in the striatum of $\Delta(9)$-tetrahydrocannabinoltolerant rats. J. Neurochem. 74, 1627-1635 (2000).

20. Giuffrida, A. et al. Dopamine activation of endogenous cannabinoid signaling in dorsal striatum. Nature Neurosci. 2, 358-363 (1999).

21. Mascia, M.S. et al. Lack of morphine-induced dopamine release in the nucleus accumbens of cannabinoid CB1 receptor knockout mice. Eur. J. Pharmacol. 383, R1-R2 (1999).

22. Comer, S.D., Lac, S.T., Curtis, L.K. \& Carroll, M.E. Effects of buprenorphine and naltrexone on reinstatement of cocaine- reinforced responding in rats. J. Pharmacol. Exp. Ther. 267, 1470-1477 (1993).

23. Landsman, R.S., Burkey, T.H., Consroe, P., Roeske, W.R. \& Yamamura, H.I. SR141716A is an inverse agonist at the human cannabinoid CB1 receptor. Eur. /. Pharm. 334, R1-R2 (1997).

24. Huestis, M.A. et al. Blockade of effects of smoked marijuana by the CB1-selective cannabinoid receptor antagonist SR141716. Arch. Gen. Psych. 58, 322-328 (2001).

25. Pilla, M. et al. Selective inhibition of cocaine-seeking behavior by a partial dopamine D-3 receptor agonist. Nature 400, 371-375 (1999).

26. Grimm, J.W. \& See, R.E. Dissociation of primary and secondary reward relevant limbic nuclei in an animal model of relapse. Neuropsychopharmacology 22, 473-479 (2000).

27. Self, D.W., Barnhart, W.J., Lehman, D.A. \& Nestler, E.J. Opposite modulation of cocaine-seeking behavior by D1- and D2-like dopamine receptor agonists. Science 271, 1586-1589 (1996).

28. Everitt, B.J. et al. Associative processes in addiction and reward. The role of amygdalaventral striatal subsystems. Ann. NY Acad. Sci. 877, 412-438 (1999). 